\title{
Commentary: At least eighty percent of brain grey matter is modifiable by physical activity: a review study
}

\author{
Irene Esteban-Cornejo ${ }^{1,2 *}$, Andrés Catena ${ }^{3}$, Charles H. Hillman ${ }^{1,4}$, Arthur F. Kramer ${ }^{1,5}$, \\ Kirk I. Erickson ${ }^{6}$ and Francisco B. Ortega ${ }^{2,7}$ \\ ${ }^{1}$ Center for Cognitve and Brain Health, Department of Psychology, Northeastern Unviersity, Boston, MA, United States, \\ ${ }^{2}$ PROmoting FITness and Health through Physical Activity Research Group, Department of Physical Education and Sport, \\ Faculty of Sport Sciences, University of Granada, Granada, Spain, ${ }^{3}$ Department of Experimental Psychology, Mind, Brain and \\ Behavior Research Center (CIMCYC), University of Granada, Granada, Spain, ${ }^{4}$ Department of Physical Therapy, Movement \\ \& Rehabilitation Sciences, Northeastern Unviersity, Boston, MA, United States, ${ }^{5}$ Beckman Institute, University of Illinois at \\ Urbana-Champaign, Champaign, IL, United States, ${ }^{6}$ Brain Aging \& Cognitive Health Lab, Department of Psychology, \\ University of Pittsburgh, Pittsburgh, PA, United States, ${ }^{7}$ Department of Biosciences and Nutrition, Karolinska Institutet, \\ Huddinge, Sweden
}

Keywords: exercise, physical activity, brain, hippocampus, lifespan

\section{A commentary on}

At least eighty percent of brain grey matter is modifiable by physical activity: a review study by Batouli, S. A. H., and Saba, V. (2017). Behav. Brain Res. 332, 204-217. doi: $10.1016 / j . b b r .2017 .06 .002$

\section{OPEN ACCESS}

Edited by:

Francesco Di Russo,

Foro Italico University of Rome, Italy

Reviewed by:

Joseph Firth,

Western Sydney University, Australia

*Correspondence:

Irene Esteban-Cornejo ireneesteban@ugr.es

Received: 30 March 2018 Accepted: 23 April 2018 Published: 08 May 2018

Citation:

Esteban-Cornejo I, Catena A, Hillman CH, Kramer AF, Erickson KI and Ortega FB (2018) Commentary: At least eighty percent of brain grey matter is modifiable by physical activity: a review study.

Front. Hum. Neurosci. 12:195.

doi: 10.3389/fnhum.2018.00195
The brain is one of the most complex and poorly understood organs of the human body. Understanding the function and complexity of the brain is considered as one of the main challenges for the twenty-first century. Almost a decade ago, in a landmark review, Hillman et al. concluded that there was converging evidence at the molecular, cellular, systems, and behavioral levels that physical activity is beneficial to the brain, however there were many important questions that remained unanswered (Hillman et al., 2008). Since that time, a growing number of publications have focused on this topic, requiring comprehensive reviews that help the field to better understand what is known and what remains to be known on the relationship of physical activity to the brain.

In a recent review published in Behavioral Brain Research (Batouli and Saba, 2017), Batouli and Saba concluded that "at least $80 \%$ of brain gray matter is modifiable by physical activity." While we believe that this review was well-conducted and well-intentioned, we disagree with the overall conclusion statement because as it overstates the extant findings. The authors summarized the brain areas and the total number of times that each area was reported in individual studies, and then created a single map containing these brain regions. It was the extensive map of regions that the authors used to reach their conclusion. However, such an approach may be greatly misleading. That is, the authors did not emphasize or take into account whether associations for a particular brain region were consistent across studies. In fact, only $\sim 10 \%$ of all reported regions were consistently related to physical activity across the reported studies. Specifically, in reference to their table there were only a handful of regions that were consistently reported across numerous studies including the hippocampus (14 times), three frontal cortical regions (i.e., superior frontal gyrus, 9 times; dorsal premotor cortex, 6 times; supplementary motor area, 5 times), two parietal regions (i.e., precuneus, 7 times; inferior parietal gyrus, 5 times) and one temporal region (middle temporal gyrus, 9 times) (Batouli and Saba, 2017). In formulating their conclusion, the authors simply aggregated all reported brain regions to create a single map, ignoring the heterogeneity of findings and then concluding a suspiciously high percentage of $80 \%$ of gray matter as modifiable by physical activity. In fact, the map provided in Figure 2 overlooks not only the number of times that a region 
was reported but also $t$-values, cluster $(K)$ sizes, specific coordinate locations and/or correction for multiple comparisons. For example, if a region was reported once with a $K \approx 40, t \approx 1.5$ and an uncorrected $P$-value, it was given the same weight in their map as a region that was reported several times with a $K \approx 500$, $t \approx 5.5$ and a corrected $P$-value. Accordingly, the authors were not rigorous in their analyses, which led to an overstatement of the extant findings in the literature.

Two additional concerns warrant consideration. First, the characterization of the physical activity measure/outcome used in the review was incorrect, as physical activity and physical fitness, which are different constructs, was confused. Physical activity is a behavior describing any bodily movement produced by skeletal muscles that requires energy expenditure, while physical fitness is a health-related marker describing a set of attributes (i.e., cardiorespiratory fitness, muscular strength, and speedagility) that people must achieve, which enables them to complete activities of daily living (Caspersen et al., 1985). Second, the brain morphology measures were also convoluted across the studies as they included both cortical volume and cortical thickness. By definition, cortical thickness is the distance between white matter and pial surfaces and cortical volume is a product of thickness and surface area. As such, the different brain outcomes must be considered as separate morphometric measurements. In fact, findings suggest that higher cortical volume but lower cortical thickness is related to better intelligence and these associations may fluctuate throughout the lifespan (Schnack et al., 2015).

\section{REFERENCES}

Batouli, S. A. H., and Saba, V. (2017). At least eighty percent of brain grey matter is modifiable by physical activity: a review study. Behav. Brain Res. 332, 204-217. doi: 10.1016/j.bbr.2017.06.002

Caspersen, C. J., Powell, K. E., and Christenson, G. M. (1985). Physical activity, exercise, and physical fitness: definitions and distinctions for health-related research. Public Health Rep. 100, 126-131.

Hillman, C. H., Erickson, K. I., and Kramer, A. F. (2008). Be smart, exercise your heart: exercise effects on brain and cognition. Nat. Rev. Neurosci. 9, 58-65. doi: $10.1038 / \mathrm{nrn} 2298$

Schnack, H. G., van Haren, N. E., Brouwer, R. M., Evans, A., Durston, S., Boomsma, D. I., et al. (2015). Changes in thickness and surface area of the human cortex and their relationship with
Collectively, the issues we raise point to serious inaccuracies and shortcomings that must be considered to more adequately interpret the claims made by Batouli and Saba (2017). In our view, a more appropriate conclusion would be: "several studies demonstrated that specific brain regions are susceptible to the influence of physical activity as denoted by changes in cortical volume and cortical thickness across the lifespan and within different populations. As such, it is difficult to formulate a conclusive statement regarding the total percentage of the brain that can be modified by physical activity. More studies are warranted to shed further light on this topic, specifically, in younger populations in which a markedly smaller proportion of studies exist."

\section{AUTHOR CONTRIBUTIONS}

IE-C, AC, and FO Contributed conception and design of the study. IE-C wrote the first draft of the manuscript. IE-C, AC, $\mathrm{FO}, \mathrm{CH}, \mathrm{KE}$, and $\mathrm{AK}$ Wrote sections of the manuscript. All authors contributed to manuscript revision, read and approved the submitted version.

\section{FUNDING}

IE-C is supported by a grant from the Alicia Koplowitz Foundation.

intelligence. Cereb. Cortex 25, 1608-1617. doi: 10.1093/cercor/ bht 357

Conflict of Interest Statement: The authors declare that the research was conducted in the absence of any commercial or financial relationships that could be construed as a potential conflict of interest.

Copyright () 2018 Esteban-Cornejo, Catena, Hillman, Kramer, Erickson and Ortega. This is an open-access article distributed under the terms of the Creative Commons Attribution License (CC BY). The use, distribution or reproduction in other forums is permitted, provided the original author(s) and the copyright owner are credited and that the original publication in this journal is cited, in accordance with accepted academic practice. No use, distribution or reproduction is permitted which does not comply with these terms. 